\title{
IMPLEMENTATION OF HORIZONTAL FOCUSING OPTICS AT APS*
}

\author{
V. Sajaev ${ }^{\dagger}$ and L. Emery, \\ Advanced Photon Source, Argonne National Laboratory, Argonne, IL
}

\begin{abstract}
Despite the very small electron beam emittance in thirdgeneration light sources, the horizontal size of the emitted $\mathrm{x}$-ray beam is still determined mainly by the electron beam divergence. Providing a converging electron beam in an undulator straight section can significantly decrease the spot size of the downstream x-rays, which leads to an increase in the photon flux density on the user sample. This converging optics method has been implemented at the Advanced Photon Source in one straight section for evaluation during machine studies. The optimization of the converging optics, the beam dynamics issues arising from the strongly asymmetric lattice optics, as well as the results achieved so far are discussed in the paper.
\end{abstract}

\section{INTRODUCTION}

The mission of the Advanced Photon Source (APS) is to provide extremely bright $\mathrm{x}$-rays for user operation. One of the key beam parameters defining the brightness is electron beam emittance. For the APS storage ring, the emittance is equal to $8 \mathrm{~nm} \times \mathrm{rad}$. But even with this very small emittance, the photon horizontal beam size is still dominated by the electron beam divergence. One way to obtain a significant reduction in the spot size and increase the photon flux density is to provide a converging electron beam in a straight section so that a waist of the $\mathrm{x}$-ray beam is achieved downstream of the insertion device at the desired distance [1]. This can be achieved by creating high horizontal $\beta$-function and non-zero positive $\alpha$ in the straight section. The first experiment with the converging $\beta$-function was completed at the ESRF facility in France [2]. An experimental study of the converging x-ray source is underway at the APS.

\section{PHOTON BEAM SIZE MINIMIZATION}

The photon beam size and divergence can be obtained from the convolution of the electron beam and $\mathrm{x}$-ray beam contributions. Divergence of $\mathrm{x}$-rays produced by an undulator is given by the equation

$$
\sigma_{r^{\prime}}=\frac{1}{2 \gamma} \sqrt{\frac{1+K^{2} / 2}{k N}}
$$

where $\gamma$ is the electron relativistic factor, $K$ is the undulator magnetic field strength parameter, $k$ is the harmonic number, and $N$ is the number of undulator poles.

For APS type A undulators, the $\mathrm{x}$-ray divergences range between the extremes shown in Table 1.

\footnotetext{
*Work supported by U.S. Department of Energy, Office of Basic Energy Sciences, under Contract No. W-31-109-ENG-38.

†sajaev@aps.anl.gov
}

Table 1: X-Ray Divergence for APS Type A Undulators.

\begin{tabular}{|c|c|c|c|}
\hline$K$ & $k$ & Photon energy $E(\mathrm{keV})$ & $\sigma_{r^{\prime}}$ \\
\hline 2.6 & 1 & 3.2 & 12.9 \\
\hline 0.9 & 3 & 30.0 & 4.2 \\
\hline
\end{tabular}

The horizontal emittance of the electron beam in the APS storage ring is $8 \mathrm{~nm} \times \mathrm{rad}$. A low-emittance lattice $(3.5 \mathrm{~nm} \times \mathrm{rad})$ is also under development. The horizontal divergence of the electron beam at the undulators for these two lattices is

$$
\begin{aligned}
& \sigma_{x}=22.4 \mu \mathrm{rad}(\varepsilon=8 \mathrm{~nm} \times \mathrm{rad}) \\
& \sigma_{x}=14.8 \mu \mathrm{rad}(\varepsilon=3.5 \mathrm{~nm} \times \mathrm{rad}),
\end{aligned}
$$

and it dominates the $\mathrm{x}$-ray divergence in most cases. Therefore, it makes sense to adjust the electron beam parameters to minimize photon spot size at the desired distance downstream. For the purposes of this work, we chose a distance of $60 \mathrm{~m}$ (more or less the usual distance between the radiation source and the user sample).

Table 2. Horizontal X-Ray Beam Sizes $60 \mathrm{~m}$ from the Undulator

\begin{tabular}{|c|c|c|c|}
\hline$\beta, \mathrm{m}$ & $\alpha$ & $\begin{array}{c}\sigma_{\mathrm{x}}, \mathrm{mm} \\
(\mathrm{x}-\mathrm{ray}, 3 \mathrm{keV})\end{array}$ & $\begin{array}{c}\sigma_{\mathrm{x}}, \mathrm{mm} \\
(\mathrm{x}-\mathrm{ray}, 30 \mathrm{keV})\end{array}$ \\
\hline \multicolumn{3}{|c|}{$\varepsilon=8 \mathrm{~nm} \times \mathrm{rad}$} \\
\hline 16 & 0 & 1.59 & 1.41 \\
\hline 60 & 1 & 1.04 & 0.73 \\
\hline \multicolumn{3}{|c|}{$\varepsilon=3.5 \mathrm{~nm} \times \mathrm{rad}$} \\
\hline 16 & 0 & 1.21 & 0.95 \\
\hline 60 & 1 & 0.90 & 0.52 \\
\hline
\end{tabular}

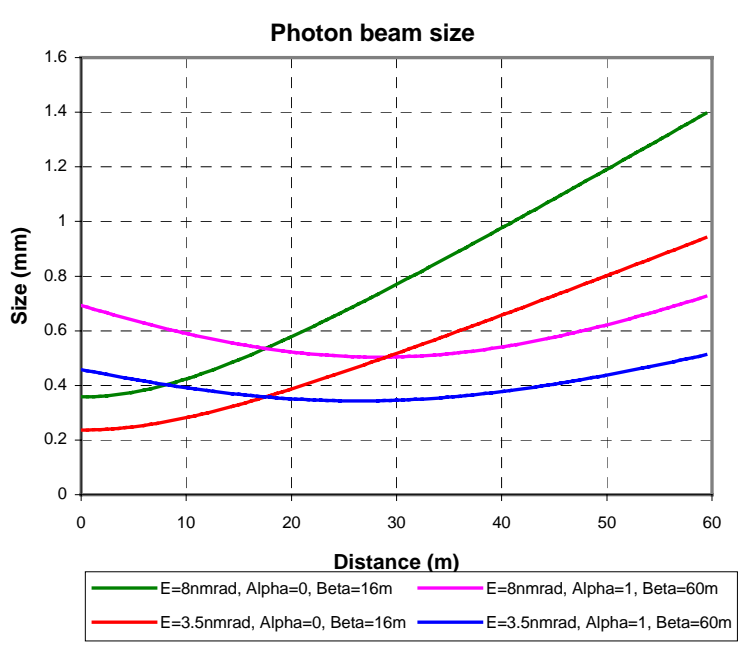

Figure 1: X-ray rms beam size as a function of distance from the undulator for the four cases presented in Table 2. 
Table 2 and Fig. 1 demonstrate that a significant reduction in $\mathrm{x}$-ray beam size can be achieved if the horizontal phase-space ellipse parameters of the electron beam can be adjusted to $\beta=60 \mathrm{~m}$ and $\alpha=1$. The choice of $\beta=60 \mathrm{~m}$ is somewhat arbitrary; to achieve a further decrease of the x-ray beam size requires a prohibitively large $\beta$ value. In the vertical plane, the electron divergence is much smaller due to small vertical emittance, and the vertical photon beam size is defined by the natural divergence of the $\mathrm{x}$-rays. That is why the behavior of the vertical $\beta$-function in the straight section is not important.

\section{LATTICE DESIGN}

The new lattice has to accommodate one straight section with the converging $\beta$-function configuration while keeping all other radiation points unchanged. Global storage ring parameters like emittance, lifetime, and injection efficiency should also be maintained.
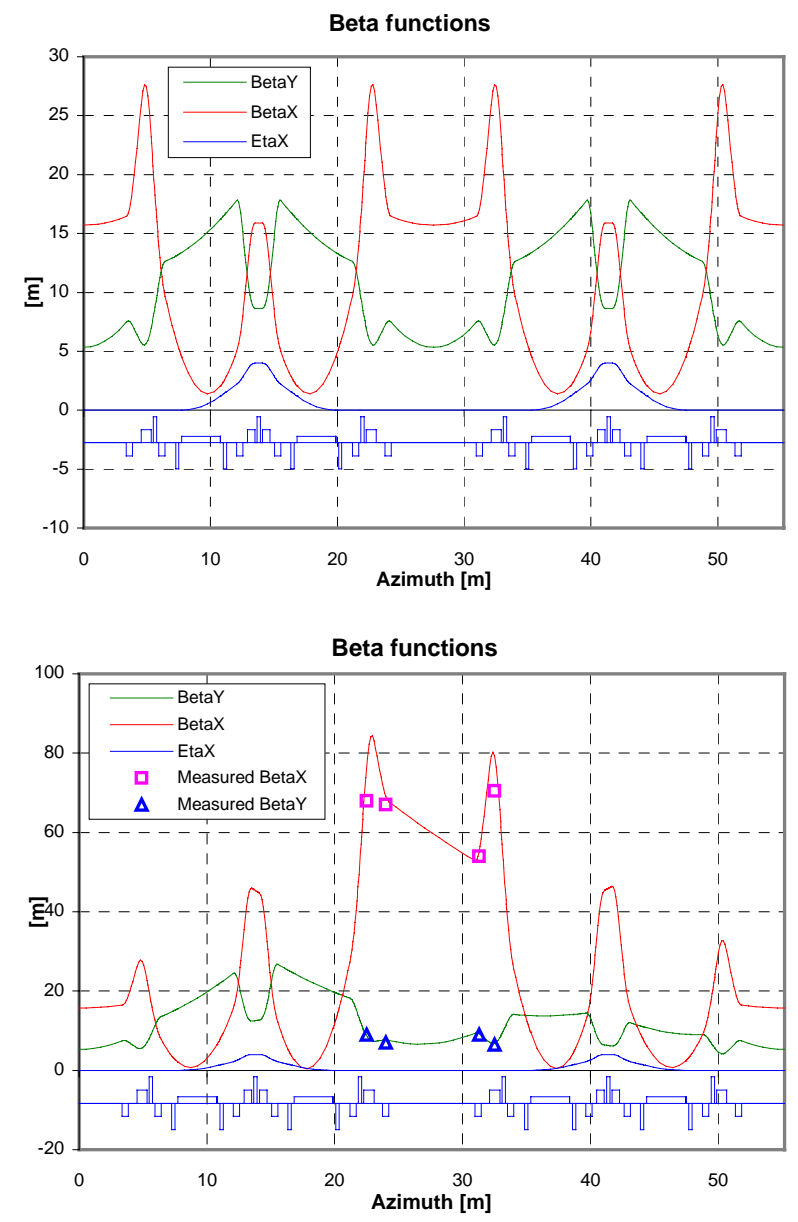

Figure 2: $\beta$-functions for sectors 35 and 36 for original APS lattice (top) and converging $\beta$-function lattice (bottom). The bottom plot also represents measured $\beta$ functions in four quadrupoles next to straight section with converging $\beta$-function.
All quadrupoles in the APS storage ring have separate power supplies, which gives great flexibility for lattice retuning. However, the power supplies are unipolar, so the sign of the quadrupole gradients cannot be changed.

The existing lattice of the APS storage ring has been modified to accommodate one straight section with the converging $\beta$-function at sector 35 . Fig. 2 shows $\beta$ functions for two consecutive sectors, 35 and 36 , for the usual APS lattice (top) and for the converging $\beta$-function lattice (bottom). The $\beta$-function in the middle of the straight section in the APS user lattice is $15 \mathrm{~m}$. The desired $\beta$-function for the converging $\beta$-function lattice is $60 \mathrm{~m}$. The difference between the existing $\beta$-function and the desired one is quite large, so the triplets in the straight section alone cannot provide it. That is why one has to use all four triplets of sectors 35 and 36 . The optical functions are fixed at the beginning of the sector 35 and at the end of sector 36, and four triplets are varied to provide the required $\beta$ and $\alpha$ in the middle of the straight section 35 . The triplets are located in the dispersion-free regions so achromatic bends are kept without changes. Finally, after the optical functions are fitted to the desired values, all storage ring triplets are used to correct for small betatron tune changes, produced by the converging $\beta$-function insertion.

High $\beta$-function values in two sectors produce strong perturbation to the harmonic sextupoles scheme used to compensate for the effect of the chromatic sextupoles and to increase the dynamic aperture. For this reason, the introduction of the high $\beta$-function straight section results in considerable decrease in the dynamic aperture. Fig. 3 (red curve) shows the dynamic aperture of the lattice with the converging $\beta$-function. A large decrease in the dynamic aperture can result in problems with the injection efficiency and with the lifetime; therefore, the sextupole scheme must be rearranged to improve the nonlinear dynamics of the ring.

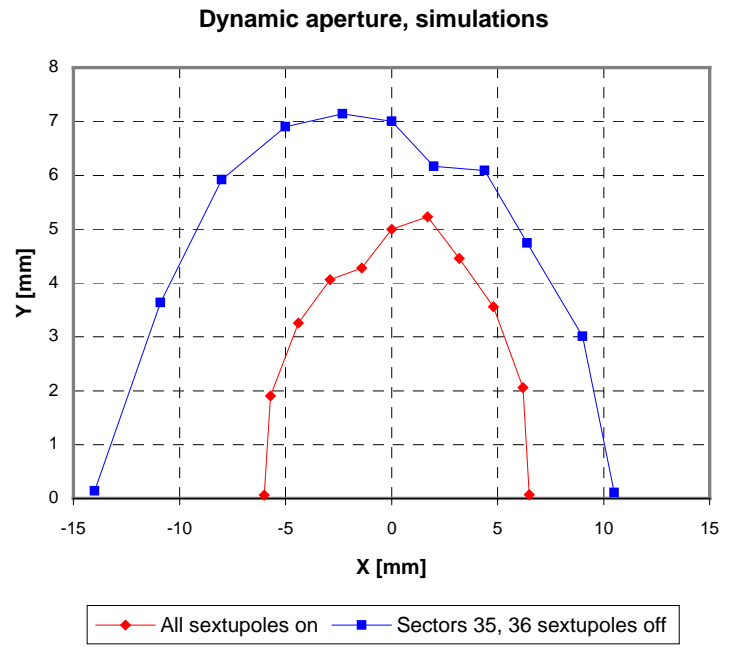

Figure 3: Dynamic aperture of storage ring with converging $\beta$-function. 
To recover the dynamic aperture, the scan over two harmonic sextupole families has been calculated. The results do not provide considerable increase in the dynamic aperture. The best result was achieved by turning off all sextupoles in the two sectors involved in the converging $\beta$-function lattice, while all other chromatic sextupoles were adjusted to correct for the chromaticity.

Fig. 3 shows the calculated dynamic aperture for the usual sextupole scheme and with the sextupoles in sectors 35 and 36 turned off. The partially recovered dynamic aperture should be enough for the injection; however, the injection efficiency may not be perfect. The dynamic aperture of the original APS lattice is much bigger than those shown on the figure and is not presented.

\section{EXPERIMENTAL RESULTS}

The experimental test of the lattice with converging $\beta$ function is underway. The designed lattice has been successfully downloaded. $\beta$-function measurements were performed by scanning quadrupole strength and measuring betatron tune change. This method allows $\beta$ functions to be measured at the quadrupole locations. Fig. 2 (bottom) presents the results of the $\beta$-function measurements together with the designed values. According to the measurements, the $\beta$-functions are close to the designed values. The $\beta$-functions at some points along the rest of the ring have also been measured. They indicated $15 \% \quad \beta$-function beating, which could be estimated as marginal. Further work is required to correct for the beating.

As expected, the emittance and the coupling of the new lattice did not change. However, the lifetime has decreased significantly.

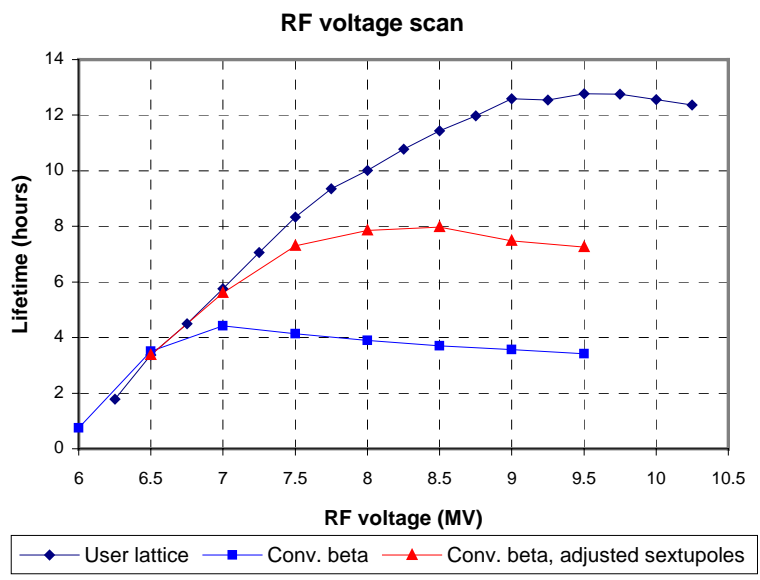

Figure 4: Lifetime vs. rf voltage scan for standard user lattice and converging $\beta$-function lattice with two different harmonic sextupole sets.

Dynamic aperture calculations showed a decrease in the nonlinear energy acceptance. To confirm that this is the reason for the lifetime decrease, the energy acceptance has been measured using rf voltage scan. Fig. 4 shows the lifetime dependence on the rf voltage for the normal user lattice and for the converging $\beta$-function optics.
The energy acceptance corresponding to the blue curve on Fig. 4 is $1.4 \%$. This is quite low for the lifetime. The harmonic sextupoles were further optimized to increase the nonlinear energy acceptance. When optimized sextupole strengths were applied to the machine, the lifetime did increase considerably. However, the injection efficiency dropped dramatically, so it was impossible to inject. Unfortunately, harmonic sextupole strengths optimized for the large energy acceptance reduced the onmomentum dynamic aperture required for the injection. This explains why the final lifetime increase due to the harmonic sextupoles rearrangement was not large.

The photon beam size decrease in the converging $\beta$ function optics was demonstrated by measuring the undulator radiation on the ID35 machine diagnostics beamline. The photon beam size was measured at the source and at the $30 \mathrm{~m}$ distance from the source. Table 3 demonstrates the beam size decrease provided by the converging $\beta$-function optics.

Table 3: Photon Beam Sizes at Different Distances from the Source.

\begin{tabular}{|l|c|c|}
\hline $\begin{array}{c}\text { Photon beam } \\
\text { size }\end{array}$ & User optics & $\begin{array}{c}\text { Converging } \beta- \\
\text { function optics }\end{array}$ \\
\hline At the source & $360 \mu \mathrm{m}$ & $\begin{array}{c}>570 \mu \mathrm{m} \text { (limited } \\
\text { by optics) }\end{array}$ \\
\hline At $30 \mathrm{~m}$ & $800 \mu \mathrm{m}$ & $560 \mu \mathrm{m}$ \\
\hline $\begin{array}{l}\text { At } 60 \mathrm{~m} \\
\text { (projected) }\end{array}$ & $1400 \mu \mathrm{m}$ & $750 \mu \mathrm{m}$ \\
\hline
\end{tabular}

\section{CONCLUSIONS}

The commissioning of the converging $\beta$-function optics has shown that considerable increase in photon flux density can be achieved by focusing the electron beam to provide the $\mathrm{x}$-ray waist downstream of the insertion device. It has also demonstrated that it is possible to break the symmetry of the APS storage ring without strong impact on performance. This finding opens up new possibilities for machine tuning to further improve the beam delivered to users.

\section{REFERENCES}

[1] E.A. Crosbie, "Focused X-Ray Sources for the APS Storage Ring," APS/IN/ACCPHY/96-1 (1996).

[2] A. Ropert, et al. "Horizontal Focusing Optics at the ESRF," EPAC2000, Vienna (June 2000). 